\title{
Traditional Chinese Medicine for Treatment of Fibromyalgia: A Systematic Review of Randomized Controlled Trials
}

\author{
Huijuan Cao, Ph.D., JianPing Liu, Ph.D., M.D., ${ }^{1,2}$ and George T. Lewith, M.A., D.M., F.R.C.P., M.R.C.G.P. ${ }^{3}$
}

\begin{abstract}
Background: Traditional Chinese Medicine (TCM) is popular for treatment of fibromyalgia (FM) although there is a lack of comprehensive evaluation of current clinical evidence for TCM's therapeutic effect and safety.

Objective: To review systematically the beneficial and harmful effects of TCM therapies for FM.

Methods: We searched six English and Chinese electronic databases for randomized clinical trials (RCTs) on TCM for treatment of FM. Two authors extracted data and assessed the trial quality independently. RevMan 5 software was used for data analyses with an effect estimate presented as mean difference (MD) with a $95 \%$ confidence interval (CI).

Results: Twenty-five RCTs were identified with 1516 participants for this review. Seven trials (28\%) were evaluated as having a low risk of bias and the remaining trials were identified as being as unclear or having a high risk of bias. Overall, ten trials were eligible for the meta-analysis, and data from remaining 15 trials were synthesized qualitatively. Acupuncture reduced the number of tender points (MD, $-3.21 ; 95 \% \mathrm{CI}-4.23$ to -2.11 ; $\mathrm{p}<0.00001, I^{2}=0 \%$ ), and pain scores compared with conventional medications (MD, $-1.78 ; 95 \% \mathrm{CI},-2.24$ to $1.32 ; \mathrm{p}<0.00001 ; I^{2}=0 \%$ ). Acupuncture showed no significant effect, with a random-effect model, compared with sham acupuncture (MD, $\left.-0.55 ; 95 \% \mathrm{CI},-1.35-0.24 ; p=0.17 ; I^{2}=69 \%\right)$, on pain reduction. A combination of acupuncture and cupping therapy was better than conventional medications for reducing pain (MD, $-1.66 ; 95 \%$ CI, -2.14 to $-1.19 ; p<0.00001 ; I^{2}=0 \%$ ), and for improving depression scores with related to FM (MD, $-4.92 ; 95 \%$ CI, -6.49 to $-3.34 ; p<0.00001 ; I^{2}=32 \%$ ). Other individual trials demonstrated positive effects of Chinese herbal medicine on pain reduction compared with conventional medications. There were no serious adverse effects reported that were related to TCM therapies in these trials.

Conclusions: TCM therapies appear to be effective for treating FM. However, further large, rigorously designed trials are warranted because of insufficient methodological rigor in the included trials.
\end{abstract}

\section{Introduction}

$\mathbf{F}$ IBROMYALGIA (FM) IS NONSPECIFIC RHEUMATISM in which typical symptoms are chronic widespread musculoskeletal pain and stiffness with accompanying fatigue, anxiety, sleep disorder; and/or irritable bowel syndrome. ${ }^{1}$ Experts in the United States and Canada have developed diagnostic criteria for identifying this disease for the American College of Rheumatology (ACR), ${ }^{2}$ which are also commonly used in China. This disease appears to affect increasing numbers of people, with a disabling outcome on their quality of life (QoL).

As the cause of FM is unknown, it is characterized and diagnosed by its symptoms of chronic widespread pain and multiple tender points associated to some degree of facilitation by the central nervous system. ${ }^{4}$ There are two important goals for treating these symptoms. ${ }^{4}$ The first goal is reduction of pain, and the second goal is restoring functionality. Many peer-reviewed articles about the treatment of FM have been published involving different pharmacologic agents including nonsteroidal anti-inflammatory drugs (NSAIDs), opioid and nonopioid analgesics, anticonvulsants, antidepressants, $\alpha$-adrenergic agonists, muscle relaxants, topical agents, local anesthetics, N-methyl-D-aspartate (NMDA) receptor antagonists, and botulinum toxin. None of these medications have proven to be effective for the entire scope of symptoms and disabilities associated with $\mathrm{FM}^{4}$ One recent systematic review ${ }^{5}$ concluded that antidepressants are associated with

\footnotetext{
${ }^{1}$ Centre for Evidence-Based Chinese Medicine, Beijing University of Chinese Medicine, Beijing, China.

${ }^{2}$ NAFKAM, University of Tromsø, Tromsø Norway.

${ }^{3}$ Department of Health Research, Complementary and Integrated Medicine Research Unit, University of Southampton, UK.

This is a full-text article of a presentation for the 5th International Congress on Complementary Medicine Research, Troms $\varnothing$, Norway, May 19-21, 2010.
} 
improvements in pain, depression, fatigue, sleep disturbances, and health-related QoL in people with FM, with large effect sizes for tricyclic and tetracyclic antidepressants (TCAs; standard mean difference $[\mathrm{SMD}]=-1.64 ; 95 \%$ confidence interval $[\mathrm{CI}],-2.57$ to -0.71$)$. Uceyler et al. ${ }^{6}$ came to similar conclusions about amitriptyline. Tofferi et al. ${ }^{7}$ suggested that cyclobenzaprine offered some benefit to patients with FM, with 1 of 5 patients experiencing improvements, while Moore et al. ${ }^{8}$ showed that pregabalin had proven efficacy for relieving neuropathic pain and FM, although many patients experienced trivial benefit or discontinued treatment because of adverse effects.

Antidepressants have been shown to have some effect, whereas NSAIDs, steroids, and calcitonin have not. Cyclobenzaprine, alprazolam, tramadol, and SAM-e have shown some benefit to patients in small studies. ${ }^{7}$ Many medications produce significant sedation and physical dependence as well as causing psychologic dependence. ${ }^{4}$

A systematic review ${ }^{9}$ of randomized clinical trials (RCTs) of nonpharmacologic interventions concluded that, although significant differences between groups occurred, the varying combinations of intervention in the studies studies and the wide range of outcome measures used made it hard to form clear conclusions across studies. The interventions included education, relaxation therapy, cognitive-behavioral therapy, acupuncture, and hydrotherapy, and there was some preliminary support for aerobic exercise.

In Traditional Chinese Medicine (TCM) theory, FM is mainly caused by emotional upsets, which affect the Liver. Stagnation of $q i$ activity leads to the stasis of Blood, which causes pain. The principle of treatment is regulating the $q i$ and Blood, combined with dispelling Cold and removing Damp. ${ }^{10}$ TCM is a whole system that uses a range of therapies to treat FM; these include acupuncture, moxibustion, herbal medicine, and massage. Two systematic reviews have suggested that acupuncture alone is effective for treating FM; one analysis involved three RCTs and four cohort studies and the other analysis included three positive RCTs and two negative RCTs. ${ }^{11,12}$ No systematic review reported the effect of herbal medicine or other TCM therapies for FM, but a review published in $2005^{13}$ suggested that "many of the herbs and other dietary supplements used by our patients are known to have potential adverse effects and may pose a risk to the patient." This current systematic review aims to update the evidence from RCTs to evaluate the therapeutic effect and safety of TCM, including Chinese herbal medicine for FM.

\section{Methods}

\section{Inclusion Criteria}

We included parallel-group RCTs of any kind of TCM treatment, including acupuncture, herbal medicine, massage, and/or cupping compared with no treatment, placebo, and/or conventional medication in patients with FM. We also included combined therapy with TCM and other interventions compared with other interventions in RCTs, or combined therapy of two kinds of TCM compared with medication or other interventions. FM was diagnosed according to recognized criteria. Outcome measures included reduction in severity of pain or depression, improvement of QoL, and reduction of relapse rate. When multiple publications reported the same groups of participants, we only included the primary publication and excluded the duplicated publications. There was no limitation on language and publication type.

\section{Identification and selection of studies}

We searched the China Network Knowledge Infrastructure (CNKI; 1979-2009), Chinese Scientific Journal Database VIP (1989-2009), Wan Fang Database (1985-2009), Chinese Biomedicine (CBM) database (1978-2009), PubMed (1966-2009) and Cochrane Library (Issue 3, 2009). All searches ended at August 2009. The search terms included fibromyalgia, fibrosis, fibrositis, myofascitis, ormyofibrositis, combined with traditional Chinese medicine, TCM, herbal, acupuncture, massage, cupping, or Tui Na. Two authors (Cao $\mathrm{H}$ and Liu JP) selected studies for eligibility and checked against the inclusion criteria independently.

\section{Data extraction and quality assessment}

Two authors (Cao H and Liu JP) extracted the data from the included trials independently. The methodological quality for RCTs was assessed, using criteria from the Cochrane Handbook for Systematic Reviews of Interventions, Version 5.0.1. ${ }^{14}$ The quality of trials was categorized into low risk of bias, unclear risk of bias, or high risk of bias according to the risk for each important outcome within included trials, including adequacy of generation of the allocation sequence, allocation concealment, blinding, whether there were incomplete outcome data ("Describe the completeness of outcome data for each main outcome, including attrition and exclusions from the analysis. State whether attrition and exclusions were reported, the numbers in each intervention group [compared with total randomized participants], reasons for attrition/exclusions where reported, and any reinclusions in analyses performed by the review authors"), ${ }^{14}$ or selective outcome, or other sources of bias.

\section{Data analysis}

Data were summarized using risk ratio $(R R)$ with $95 \% \mathrm{CIs}$ for binary outcomes or mean difference (MD) with a $95 \% \mathrm{CI}$ for continuous outcomes. We used Revman 5.0.2 software from the Cochrane Collaboration for data analyses. Metaanalysis was used if the trials had acceptable homogeneity of study design, participants, interventions, controls, and outcome measures. Statistical heterogeneity was tested by examining $I^{2}$ square ${ }^{15}$ or $p$-value; an $I^{2}>50 \%$ or a $p$-value $<0.1$ indicates the possibility of statistical heterogeneity. Both a fixed-effect model and a random-effects model were used if there was a possibility of statistical heterogeneity among the trials. If the $I^{2}$ was $<50 \%$, or the $p$-value was $>0.1$, only a fixed-effect model was used for meta-analysis. Publication bias was explored via a funnel-plot analysis.

\section{Results}

\section{Description of studies}

After primary searches in six databases, 883 citations were identified.The majority was excluded because of obvious ineligibility, and full-text papers of 35 studies were retrieved. Finally, 25 $5^{16-38}$ randomized trials were included in this review, two trials ${ }^{39,40}$ were excluded as duplicated publications, and eight trials ${ }^{41-48}$ were excluded because the 


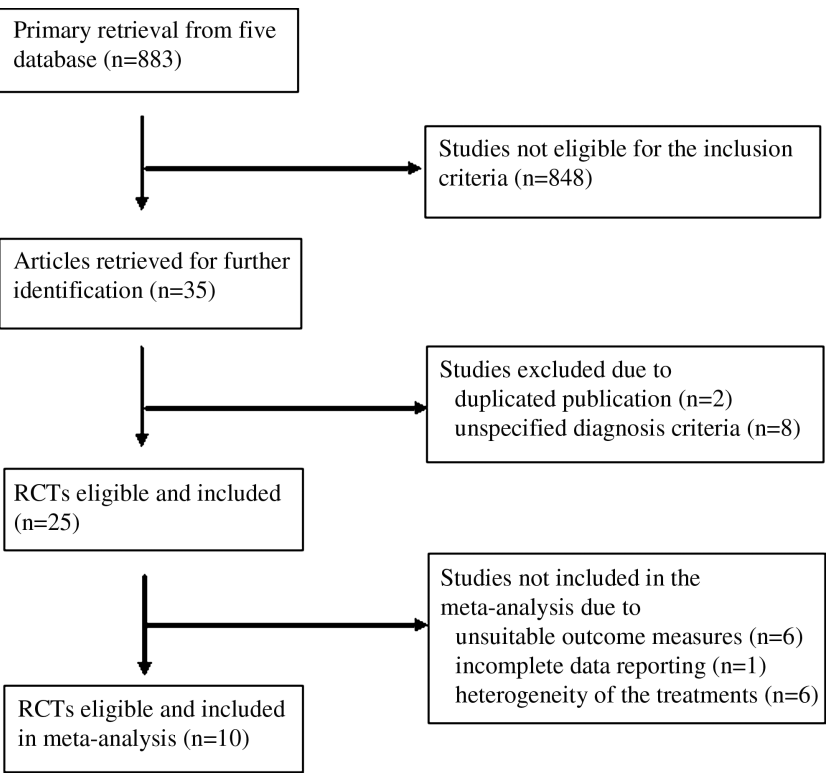

FIG. 1. Study enrollment process. RCTs, randomized controlled trials.

diagnosis criteria were not specified (Fig. 1). The characteristics of included trials are listed in Table 1. Of the included trials, two were unpublished postgraduate student dissertations. $^{*, \dagger}$

The twenty-five RCTs involved a total of 1516 patients with FM. The participants were ages 17-77, and the disease duration was from 3 months to 20 years. Twenty-three trials used ACR 1990 as the diagnostic criteria, one trial ${ }^{31}$ used the International Academy of Soreness Research (IASR) ${ }^{53}$ for diagnosing FM, and one trial ${ }^{26}$ made diagnoses according to the subjects' symptoms. The interventions included acupuncture (electroacupuncture, auricular acupuncture), cupping, herbal medicine (decoctions, capsules, and external preparations), massage, moxibustion, and combinations of acupuncture and cupping, or acupuncture and herbal medicine. The controls included no treatment, sham acupuncture, or conventional medications. The treatment duration ranged from 12 days to 12 weeks. Changes in visual analogue scale (VAS) scores as the major outcome measurement were reported in 15 trials. Seven trials ${ }^{18,23,27,31,33,34, \dagger}$ calculated the change of number of tender points, and used the McGill Pain Questionnaire (MPQ), Present Pain Intensity (PPI), or Fibromyalgia Impact Questionnaire (FIQ) for assessing intensity of pain. Four trials ${ }^{20,25,28, *}$ used the Hamilton Depression Scale (HAMD) or Hamilton Anxiety Scale (HAMA) to assess depression or anxiety. Three trials ${ }^{16,24,34}$ used the ShortForm-36 (SF-36) and QoL scale (QoLS) for measuring QoL. Six trials used four categories to evaluate treatment effects

*Fu XY, Li CD. Clinical randomized controlled trial on combination of acupuncture, cupping and medicine for treatment of fibromyalgia syndrome [dissertation for Master's degree from Chengdu University of Traditional Chinese Medicine, Chengdu, China]. 2004.

'Guo Y, Sun YZ. Clinical observation of therapeutic effect of penetration needling at the back in treating with fibromyalgia syndrome [dissertation for Master's degree from Heilongjiang University of Traditional Chinese Medicine, Heilongjiang, China]. 2005. including cure (symptoms disappeared and no tender points existed), markedly effective (symptoms reduced more than $>50 \%$ ), effective/reduced (symptoms reduced between $25 \%$ and $50 \%$ ), and ineffective (symptoms reduced $<25 \%$ ). The combined rates of the markedly effective and effective treatments were used to calculate a total effective rate, which was the main outcome measure in some trials.

\section{Methodological quality}

According to our predefined quality assessment criteria, seven $16,19,24,28,32,34, \dagger$ of the 25 trials (28\%) were evaluated as having a low risk of bias, and another 18 included trials were evaluated as having an unclear risk of bias (Table 2). The sample size varied from 10 to 38 participants, with an average of 25 patients per group. None of the trials reported prior sample-size calculation, 11 trials ${ }^{16,19,21,24,25,28,32,34,35,37}$ described randomization procedures (using a random number table, computer generation of random numbers, or a drawing), but only two trials ${ }^{19,24}$ reported adequate allocation concealment. Four trials ${ }^{19,24,32,34}$ blinded both patients and outcome assessors, one trial ${ }^{16}$ only blinded patients, and three trials $28,33, *$ blinded the outcome assessors. Nine trials $^{16,17,19,21,24,28,32,34, *}$ reported the number of dropouts, and three trials ${ }^{16,32,34}$ used intention-to-treat analysis.

\section{Effect estimates (Table 3)}

Therapeutic effect of acupuncture. Twelve trials ${ }^{16,19}$, $22-24,26,31-34,38, \dagger$ tested acupuncture for treating FM. Six trials $16,19,24,26,32,33$ compared acupuncture or electroacupuncture with sham acupuncture or sham electroacupuncture, and another six trials $22,23,31,34,38, \dagger$ compared acupuncture with conventional medications.

A pooling analysis of four trials ${ }^{16,19,24,32}$ showed a significant effect of acupuncture compared to sham acupuncture for reducing pain according to VAS scores after treatment (MD, $-1.24 ; 95 \% \mathrm{CI},-1.47$ to $-1.01 ; \mathrm{p}<0.00001)$. However, this effect became nonsignificant (MD, -0.55 ; 95\% CI, -1.35$0.24, p=0.17)$ when using a random-effects model because of significant heterogeneity $\left(I^{2}=69 \%\right)$. Three trials ${ }^{23,31, \dagger}$ compared acupuncture with conventional medications, and the pooling results showed that acupuncture was significantly better than conventional medications for reducing pain (MD, $-1.78 ; 95 \%$ CI -2.24 to $-1.32, p<0.00001 ; I^{2}=0 \%$ ) and number of tender points (MD, $-3.21 ; 95 \% \mathrm{CI},-4.23$ to -2.11 ; $\left.p<0.00001 ; I^{2}=0 \%\right)$.

Two trials ${ }^{22,23}$ reported the relapse rate after 6 months, suggesting that acupuncture (including transcutaneous electrical nerve stimulation [TENS] and electroacupuncture) was significantly better than amitriptyline for preventing relapse ( $R R$ 0.28; 95\% CI, 0.11-0.67; $p=0.005 ; I^{2}=0 \%$ ).

Data from four trials $26,33,34,38$ were not included in the meta-analysis because of poor reporting. The main findings of those trials are presented in Table 4.

As a result of the insufficient number of included trials in one meta-analysis, a meaningful funnel plot analysis was not feasible.

Therapeutic effect of herbal medicine. Six trials $^{20,21,25,30,36,37}$ tested herbal medicine against Western medicine for FM. Three trials ${ }^{20,36,37}$ tested herbal decoctions, two trials ${ }^{25,30}$ tested a Chinese patent medicine, and one trial ${ }^{21}$ 


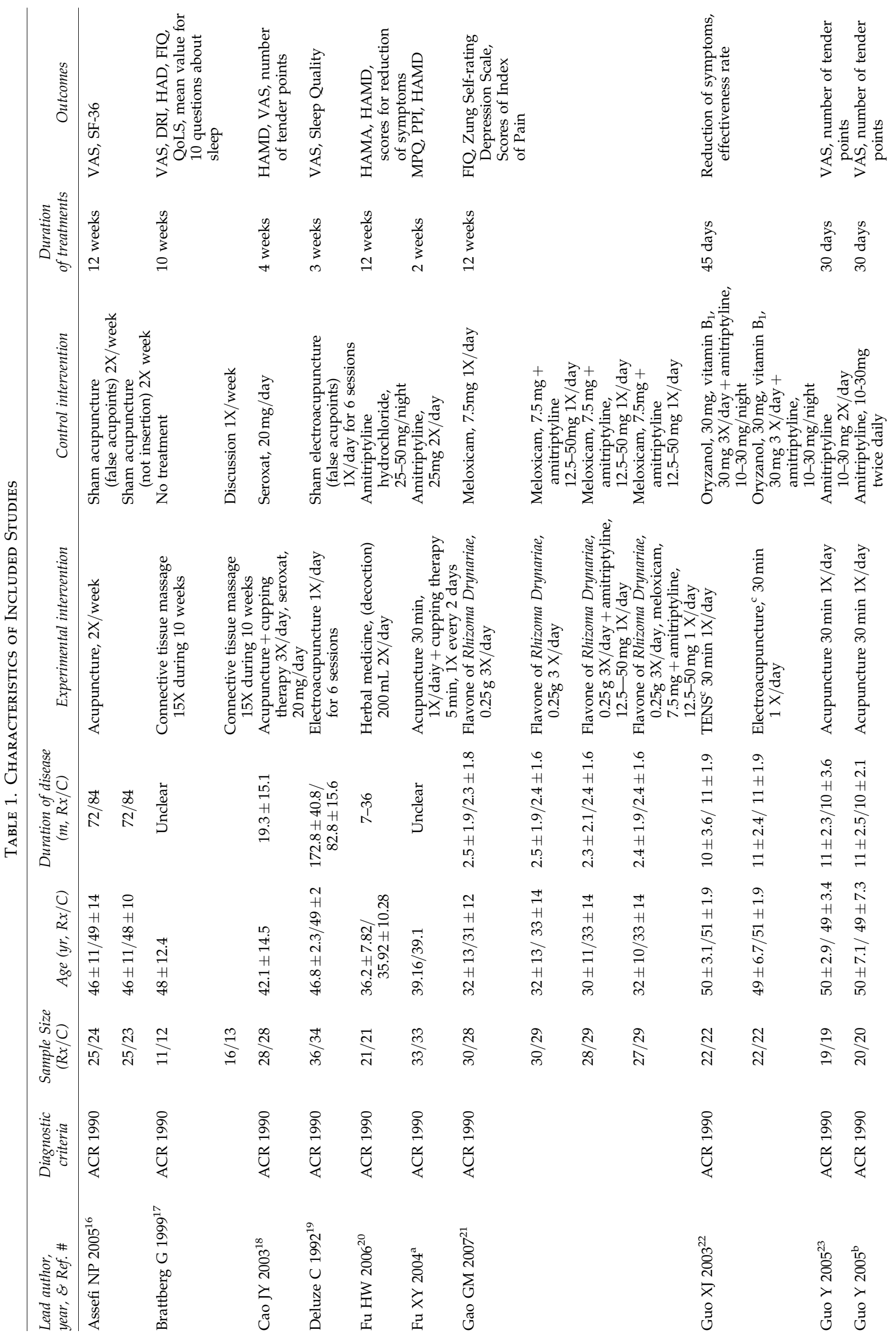



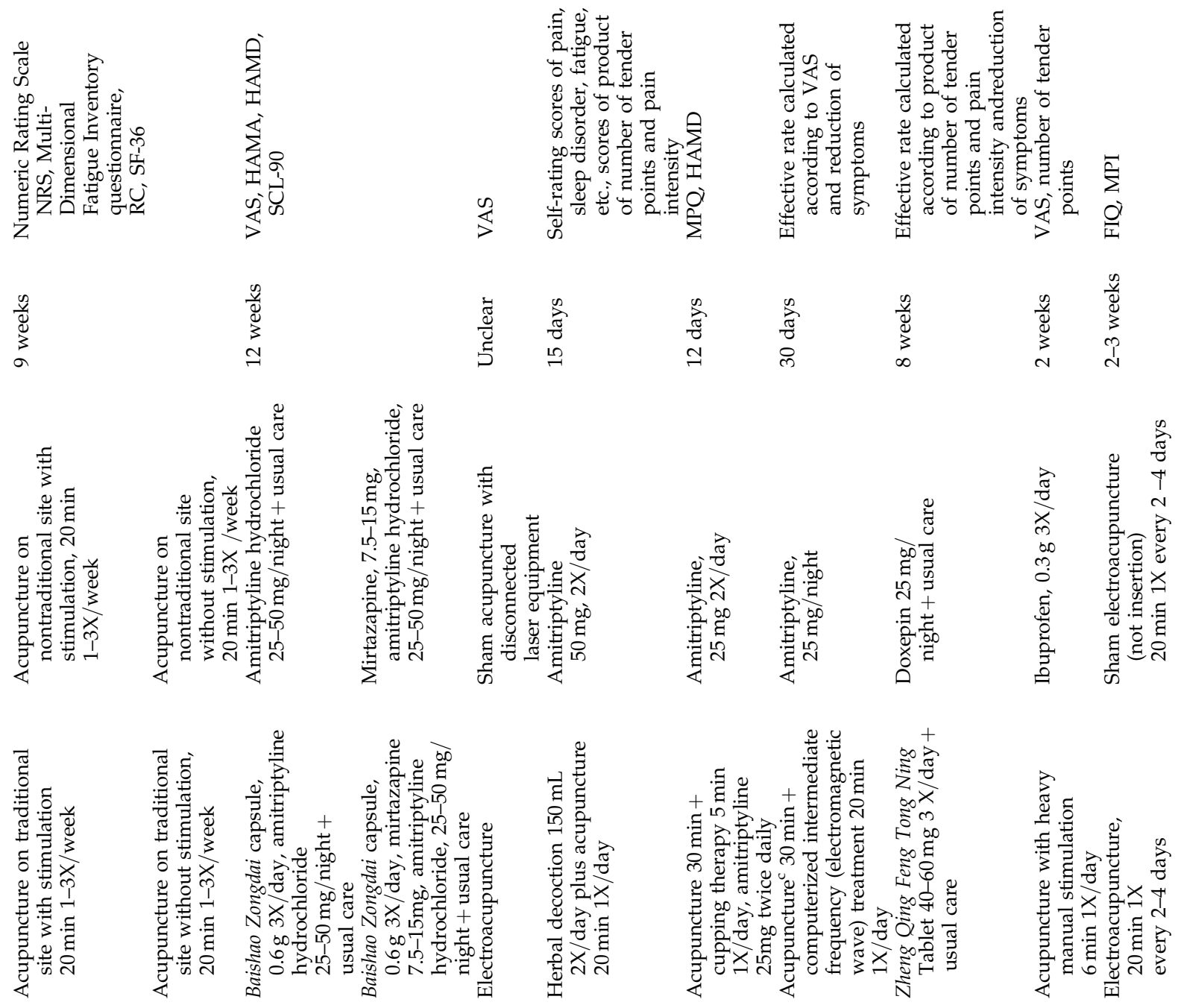

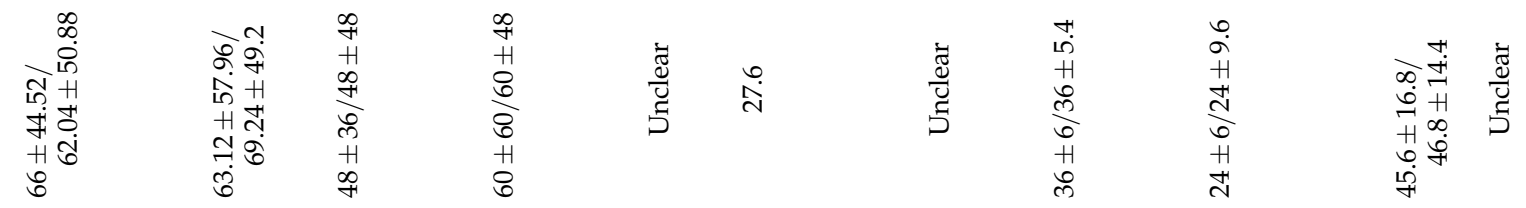

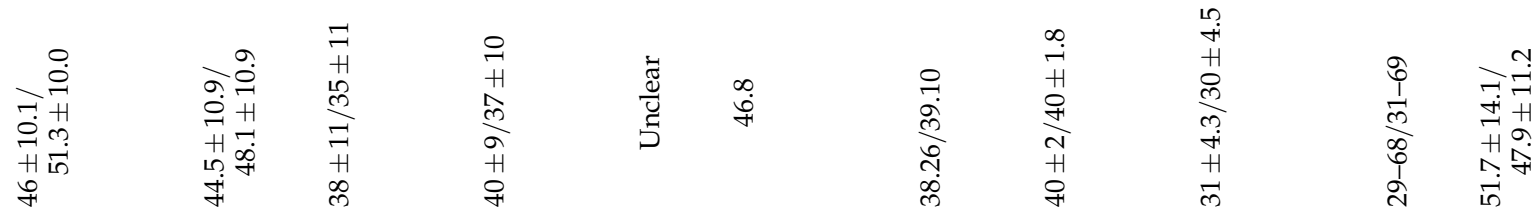

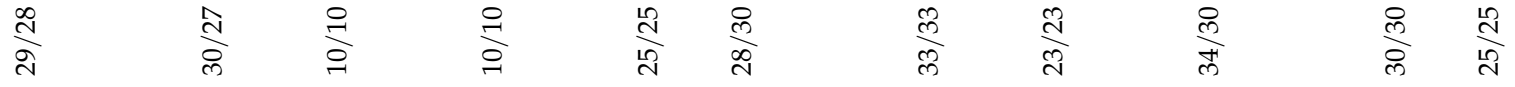

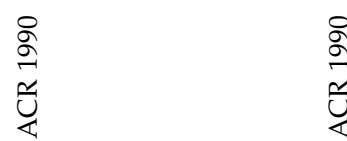

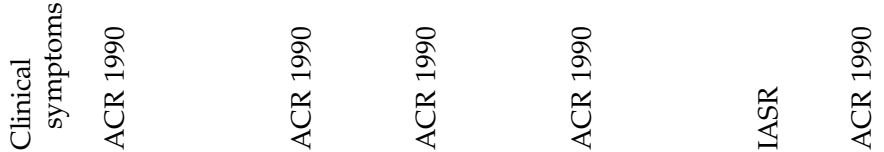

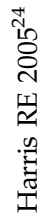

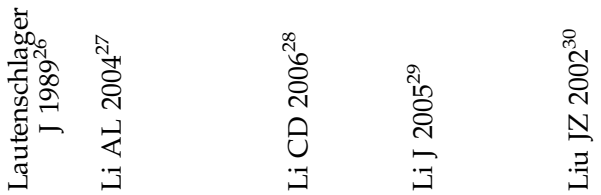

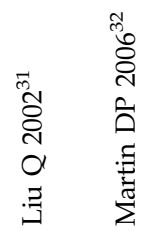




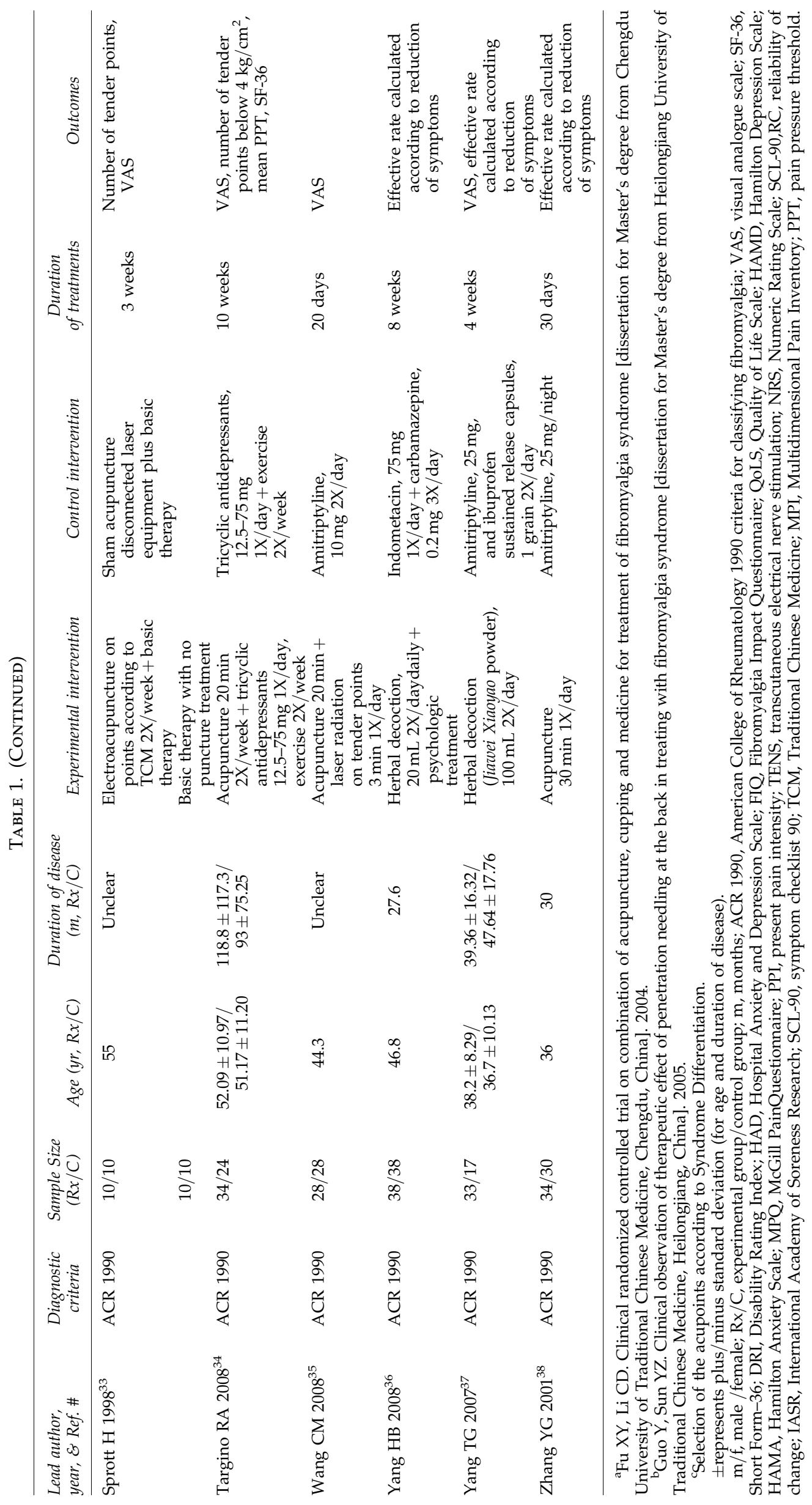




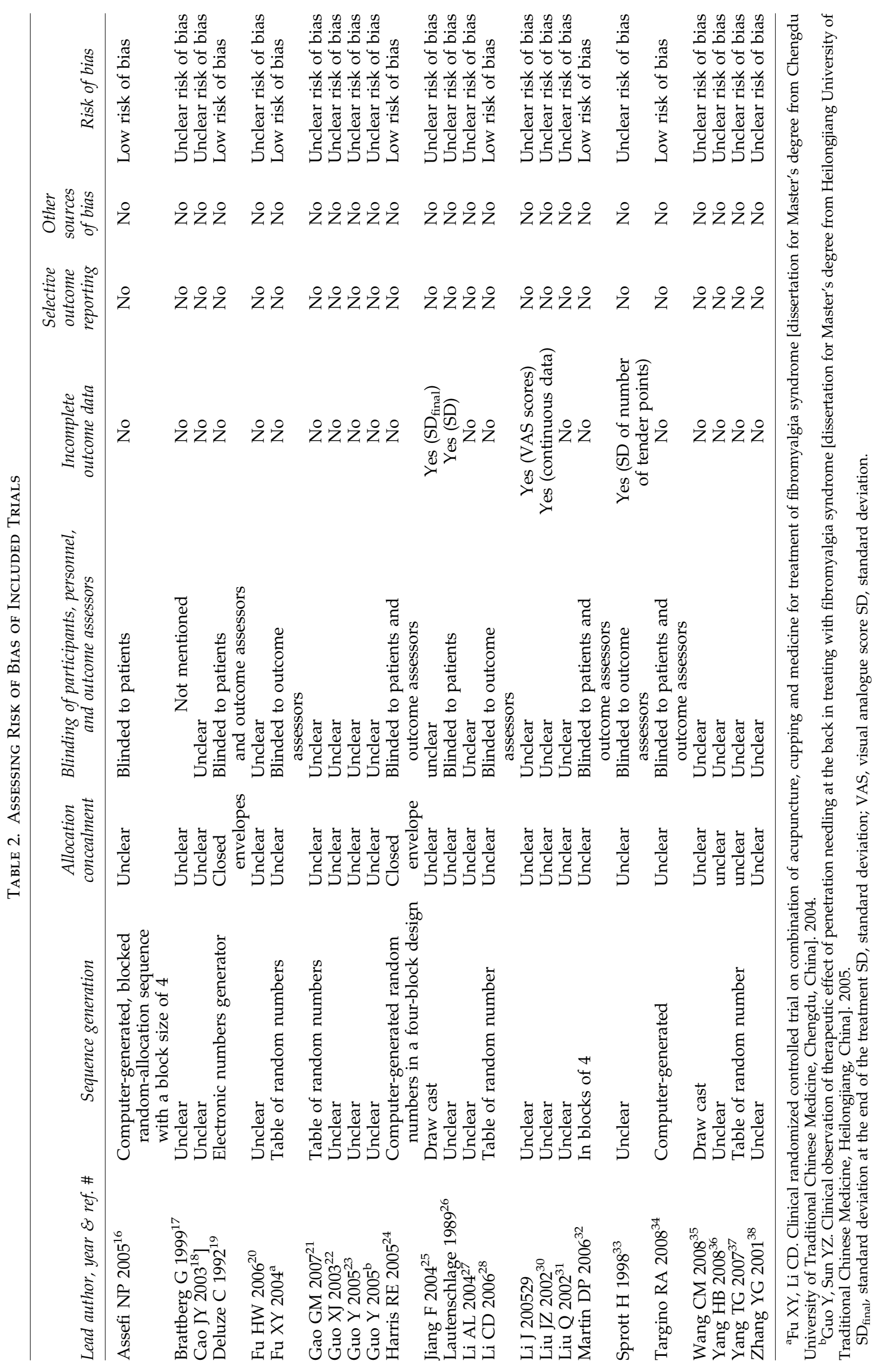


Table 3. Estimated Effect of Included Trials in Meta-Analyses

\begin{tabular}{|c|c|c|c|c|}
\hline Lead author, year and ref. \# & Interventions & Estimate effects & Weight & p-value \\
\hline \multicolumn{5}{|c|}{ 1. VAS scores after treatment } \\
\hline \multicolumn{5}{|c|}{ 1.1 Therapeutic effect of acupuncture } \\
\hline \multicolumn{5}{|c|}{ 1.1.1 Acupuncture versus sham acupuncture } \\
\hline Assefi NP $2005^{16}$ & $\begin{array}{l}\text { Acupuncture versus sham acupuncture } \\
\text { on false acupoints }\end{array}$ & $0.10[-1.19,1.59]$ & $13.67 \%$ & \\
\hline Assefi NP $2005^{16}$ & $\begin{array}{l}\text { Acupuncture versus sham acupuncture } \\
\text { without insertion }\end{array}$ & $-0.30[-1.64,1.04]$ & $13.18 \%$ & \\
\hline Deluze C $1992^{19}$ & $\begin{array}{l}\text { Electroacupuncture versus sham elec- } \\
\text { troacupuncture on false acupoints }\end{array}$ & $-1.39[-1.64,-1.14]$ & $35.09 \%$ & \\
\hline Harris RE $2005^{24}$ & $\begin{array}{l}\text { Acupuncture on traditional site versus } \\
\text { acupuncture on nontraditional site }\end{array}$ & $-1.30[-2.07,0.10]$ & $13.01 \%$ & \\
\hline Harris RE $2005^{24}$ & $\begin{array}{l}\text { Acupuncture on traditional site with } \\
\text { stimulation versus acupuncture on } \\
\text { nontraditional site with stimulation }\end{array}$ & $0.92[-0.59,2.43]$ & $11.70 \%$ & \\
\hline \multirow[t]{3}{*}{ Martin DP $2006^{32}$} & $\begin{array}{l}\text { Electroacupuncture versus sham elec- } \\
\text { troacupuncture without insertion }\end{array}$ & $-0.70[-2.06,0.66]$ & $13.34 \%$ & \\
\hline & Overall (FEM, $\left.I^{2}=69 \%\right)$ & MD -1.24 [-1.47, -1.01] & $100 \%$ & $<0.00001$ \\
\hline & Overall (REM, $\left.I^{2}=69 \%\right)$ & MD -0.55 [-1.35, 0.24] & $100 \%$ & 0.17 \\
\hline \multicolumn{5}{|c|}{ 1.1.2 Acupuncture versus conventional medications } \\
\hline Guo Y $2005^{23}$ & Acupuncture versus amitriptyline & $-1.71-2.39,-1.03]$ & $39.69 \%$ & \\
\hline Guo Y $2005^{\mathrm{a}}$ & Acupuncture versus amitriptyline & $-1.66[-2.97,-0.35]$ & $21.63 \%$ & \\
\hline \multirow[t]{2}{*}{ Liu Q $2002^{31}$} & Acupuncture versus ibuprofen & $-1.90[-2.61,-1.19]$ & $38.68 \%$ & \\
\hline & Overall (FEM, $\left.I^{2}=0 \%\right)$ & MD -1.78 [-2.24, -1.32] & $100 \%$ & $<0.00001$ \\
\hline \multicolumn{5}{|c|}{ 1.2 Combination of acupuncture and cupping therapy + conventional medications versus medications alone } \\
\hline Cao JY $2003^{18}$ & $\begin{array}{l}\text { Acupuncture + cupping therapy and } \\
\text { seroxat versus seroxat alone }\end{array}$ & $-1.63[-2.18,-1.08]$ & $44.62 \%$ & \\
\hline \multirow[t]{2}{*}{ Li CD $2006^{28}$} & $\begin{array}{l}\text { Acupuncture + cupping therapy and } \\
\text { amitriptyline versus amitriptyline }\end{array}$ & $-1.77[-2.74,0.80]$ & $55.38 \%$ & \\
\hline & Overall (FEM, $\left.I^{2}=0 \%\right)$ & MD -1.66 [-2.14, 1.19] & $100 \%$ & $<0.00001$ \\
\hline \multicolumn{5}{|c|}{$\begin{array}{l}\text { 2. \# of tender points after treatment } \\
\text { Therapeutic effect of acupuncture }\end{array}$} \\
\hline Guo Y $2005^{23}$ & Acupuncture versus amitriptyline & $-4.00[-6.73,-1.27]$ & $16.4 \%$ & \\
\hline Guo Y $2005^{\mathrm{a}}$ & Acupuncture versus amitriptyline & $-3.30[-6.02,-0.58]$ & $16.5 \%$ & \\
\hline \multirow[t]{2}{*}{ Liu Q $2002^{31}$} & Acupuncture versus ibuprofen & $-3.00[-4.35,-1.65]$ & $67.1 \%$ & \\
\hline & Overall (FEM, $\left.I^{2}=0 \%\right)$ & $\mathrm{MD}-3.21[-4.23,-2.11]$ & $100 \%$ & $<0.00001$ \\
\hline
\end{tabular}

\section{HAMD scores after treatment}

Combination of acupuncture and cupping therapy + conventional medications versus conventional medications alone

Cao JY $2003^{18} \quad$ Acupuncture plus cupping therapy and $\quad-6.00[-8.36,-3.64] \quad 44.62 \%$

Li CD $2006^{28}$ seroxat versus seroxat alone

Acupuncture plus cupping therapy and $\quad-4.04[-6.16,-1.92] \quad 55.38 \%$ amitriptyline versus amitriptyline

Overall (FEM, $\left.I^{2}=32 \%\right) \quad$ MD $-4.92[-6.49,-3.34] \quad 100 \% \quad<0.00001$

4. Relapse rate after 6 months of treatment

Acupuncture versus conventional medications

Guo XJ $2003^{22}$

Guo XJ $2003^{22}$

Guo Y $2005^{23}$
TENS versus oryzanol, vitamin $\mathrm{B}_{1}$, and amitriptyline

Electroacupuncture versus oryzanol, vitamin $B_{1}$ and amitriptyline

Acupuncture versus amitriptyline

$\begin{array}{lr}0.30[0.06,1.44] & 32.6 \% \\ 0.30[0.06,1.44] & 32.6 \% \\ & \\ 0.24[0.05,1.03] & 34.7 \% \\ \mathbf{0 . 2 8}[\mathbf{0 . 1 1}, \mathbf{0 . 6 7}] & \mathbf{1 0 0 \%}\end{array}$

Overall (FEM, $\left.I^{2}=0 \%\right)$

${ }^{\mathrm{a}}$ Guo $\mathrm{Y}$, Sun YZ. Clinical observation of therapeutic effect of penetration needling at the back in treating with fibromyalgia syndrome [dissertation for Master's degree from Heilongjiang University of Traditional Chinese Medicine, Heilongiiang, China]. 2005.

VAS, visual analogue scale; FEM, fixed effect model; MD, mean difference; REM, random effect model; HAMD; Hamilton Depression Scale; TENS, transcutaneous electrical nerve stimulation; $R R$, risk ratio. 
tested active components of an herbal medicine. As a result of the variation of herbal medicines, the data were not combined. The main findings of the six trials are presented in Table 4.

Therapeutic effect of massage. Only one small trial ${ }^{17}$ tested massage for treatment of FM and showed no significant difference between "connective tissue" massage and no treatment for reducing pain according to VAS scores after treatment (MD, $-0.58 ; 95 \% \mathrm{CI},-1.76-0.60 ; p=0.34$ ).

Therapeutic effect of combination therapies of TCM. Six trials ${ }^{17,27-29,35,^{*}}$ tested the therapeutic effect of combination TCM therapies for treating FM. Three trials ${ }^{18,28, *}$ compared acupuncture plus cupping therapy with medications, one trial $^{29}$ compared acupuncture plus computerized intermediate frequency treatment with amitriptyline, one trial ${ }^{35} \mathrm{com}$ pared acupuncture plus laser treatment with amitriptyline, and one trial ${ }^{27}$ compared acupuncture plus herbal medicine with Western medications.

Two trials ${ }^{18,28}$ showed that a combination of acupuncture and cupping therapy plus medications was significantly better than conventional medications alone for reducing pain (MD, $-1.66,95 \% \mathrm{CI}, 2.14$ to $-1.19, p<0.00001, I^{2}=0 \%$ ) and HAMD scores (MD, $-4.92,95 \% \mathrm{CI},-6.49$ to -3.34 , $p<0.00001, I^{2}=32 \%$ ).

Four trials ${ }^{27,29,35, *}$ were not included in the meta-analysis as a result of the data being unavailable. The main findings of these trials are presented in Table 4.

Adverse effects. An outcome of adverse events was described in 11 trials. ${ }^{16,20,21,25,27,29,30,34,36, * \dagger}$ Adverse effects from eight trials $16,21,25,29,30,34, *, \dagger$ were related to TCM therapies, including four ${ }^{16,29,34, \dagger}$ related to acupuncture, three to herbal medicine, and one* to cupping therapy. The adverse effects of acupuncture were bruising, nausea, fainting, discomfort at the sites of needle insertions or simulated needle insertions, and temporary edema of the hand. Nausea, fainting, dry mouth, bimalleolar edema, and skin rash were reported as adverse effects of herbal medicines. One trial* reported that 1 patient had mild scalding on the skin after being included in a cupping group $(n=33)$.

Lethargy, nausea, fainting, dry mouth, fatigue, blurred vision, hyperhydrosis, and constipation were reported adverse effects of conventional medications.

No serious adverse event was reported in any of the included trials.

\section{Discussion}

The data from the 25 RCTs that were analyzed demonstrate that, acupuncture, acupuncture combined with cupping therapy, or acupuncture combined with cupping and conventional medication were significantly more effective than conventional medication alone for reducing pain and number of tender points in subjects with FM. The therapeutic effect of acupuncture appears to be similar to sham acupuncture for pain reduction, but more data are needed to prove this finding. The therapeutic effects of herbal medicine and massage are uncertain due to limited numbers of clinical trials.

There are several limitations in this review. The quality of the included studies is generally poor, which indicates an unclear risk of bias resulting from insufficient reporting of methodological components in the trials. There were unclear descriptions of randomization procedures and lack of blinding in the majority of trials, which may have created potential performance biases and detection biases, as patients and researchers might have been aware of the therapeutic interventions. Intention-to-treat analysis was not applied in most of the included trials, and although it was not possible to perform a meaningful funnel-plot analysis because of the insufficient number of included trials in the meta-analysis, there remained the possible existence of publication bias. To the ability to perform meta-analysis was limited because of the heterogeneity of the interventions among the included trials and the variance of composite outcome measures used in 15 of the included trials. This was particularly relevant to the outcome classifications as cure, markedly effective, effective or ineffective, which were used in six trials and but was not validated; thus the findings were hard to interpret. Consequently, interpretation of these positive findings should be cautious, and the study methodology needs to be improved for future confirming studies.

The searches conducted in the present study identified four systematic reviews of acupuncture for treating FM. The latest one ${ }^{50}$ published in 2009 included only six trials, which were all included in this systematic review. In this systematic review five high-quality trials compared acupuncture with sham acupuncture, which may not be appropriate as a placebo against which to evaluate the therapeutic effect of real acupuncture. ${ }^{51}$ There were 12 trials evaluating acupuncture for FM in this review, but only two of them used syndromedifferentiation for acupuncture-point selection. The data suggesting that acupuncture is effective for FM should be taken as being tentative and further randomized trials are warranted. Control interventions in such trials should be carefully selected, as, at the present time, there is not a proper "acupuncture placebo."

Nine of the included trials ${ }^{16,17,22,23,25,32-34,38}$ reported the results of follow-up. One trial ${ }^{34}$ followed all the patients for 2 years, reporting that the acupuncture group was significantly better than the control group with respect to numbers of tender points after 6 months, but, at 2 years, noting that there was no significant difference in any outcomes. One trial $^{32}$ followed all patients for 7 months and showed no difference between acupuncture and sham acupuncture for pain reduction. Assefi et al. ${ }^{16}$ followed all patients for 6 months, suggesting that the observed effect was probably driven by the higher mean score in the simulated-acupuncture group compared with the sham-needling group. Another trial ${ }^{17}$ followed patients for up to 6 months and showed no statistically significant differences between massage and no treatment for the tested parameters at 3 and 6 months. Two trials ${ }^{22,23}$ followed the patients for 6 months, suggesting that acupuncture was significantly better than amitriptyline for preventing relapse after treatment, although the number of patients who were followed was too small for substantial statistical analysis. There is no evidence for a long-term effect of herbal medicine or cupping therapy.

Most of the existing trials are of small size and have an unclear risk of bias or a high risk of bias. Further rigorously designed trials are needed to confirm the effectiveness of TCM therapy for treating FM. Randomization methods need to be described clearly and reported fully. Although blinding of patients and practitioners might be very difficult for 


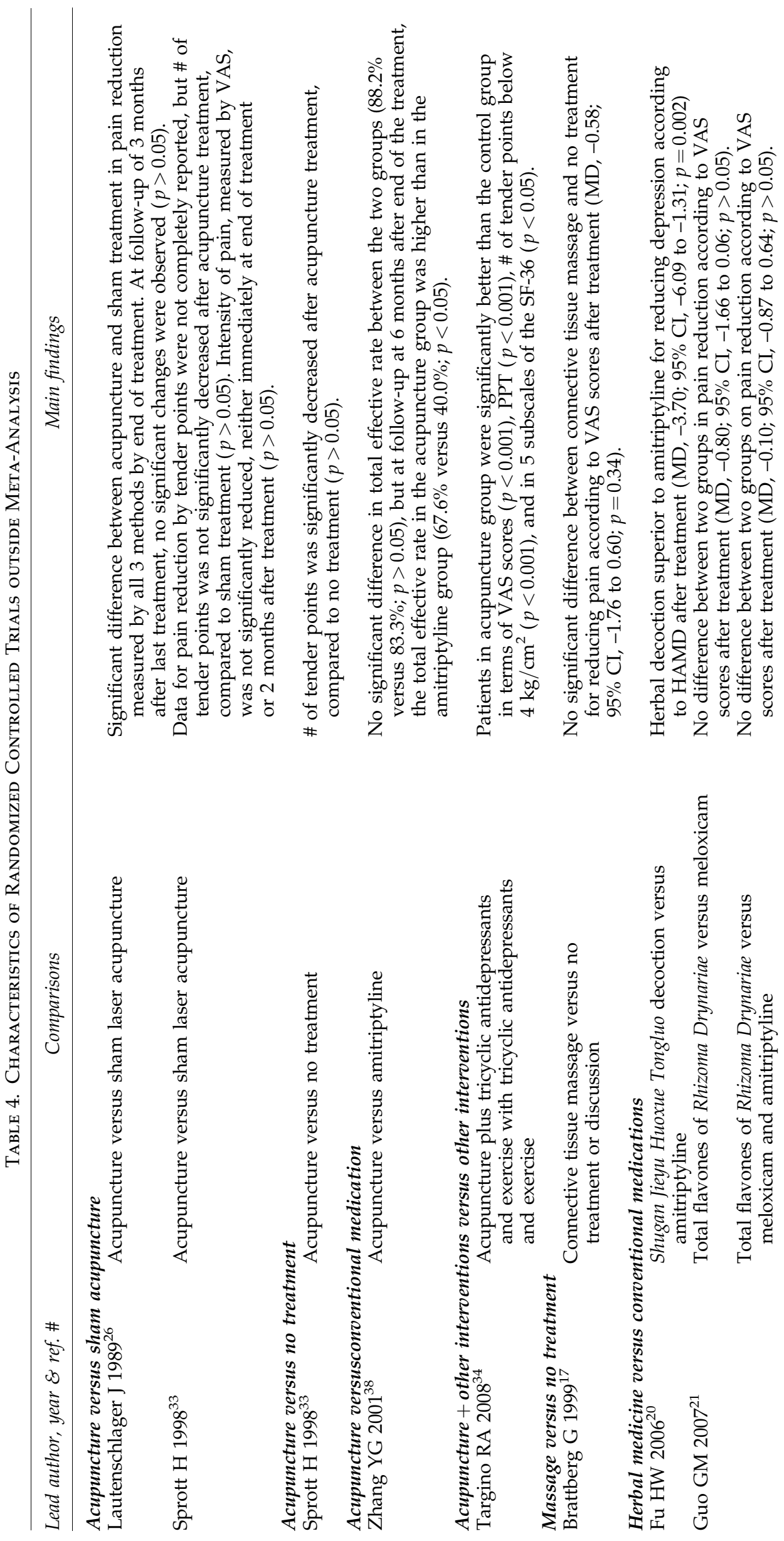



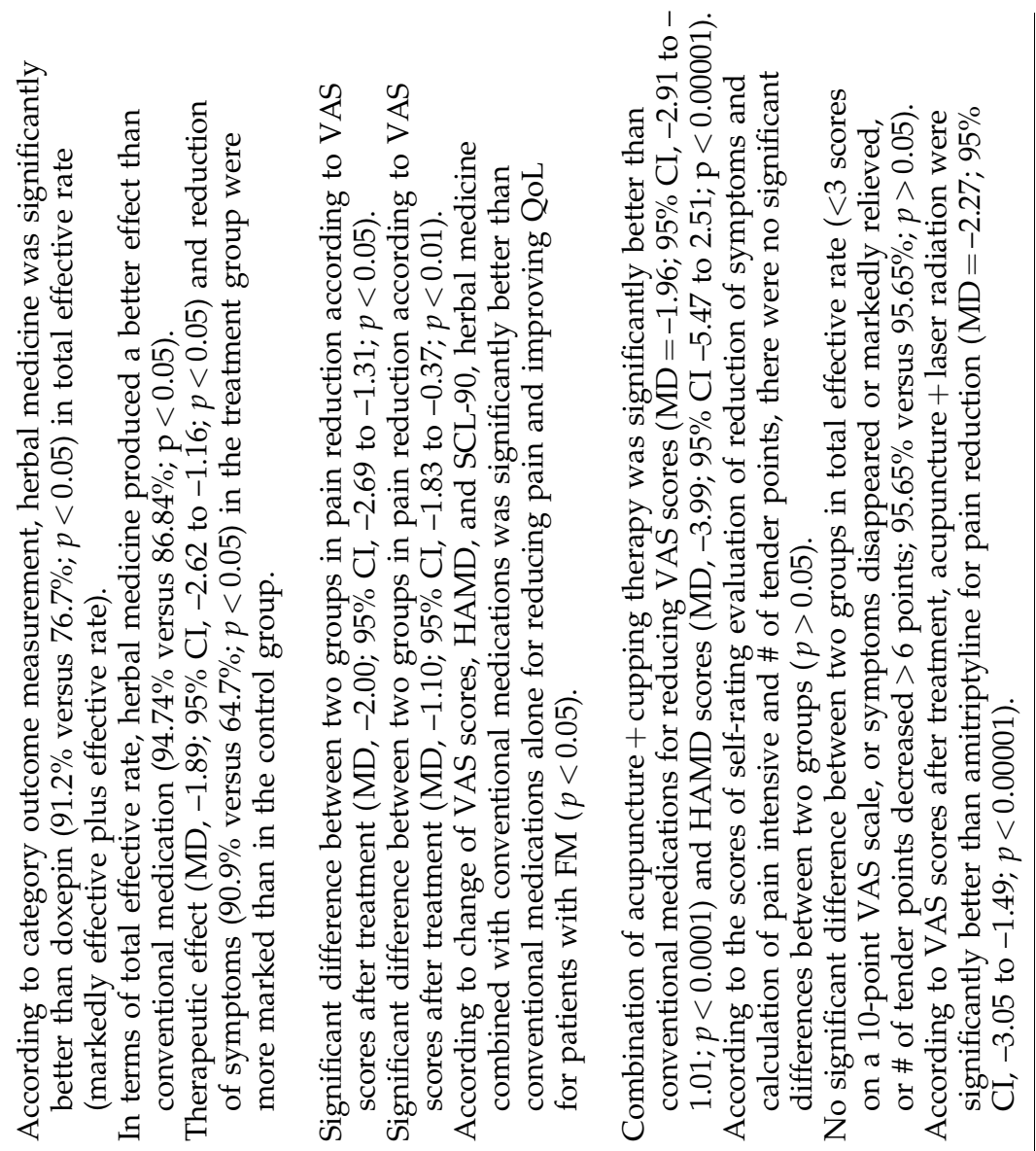

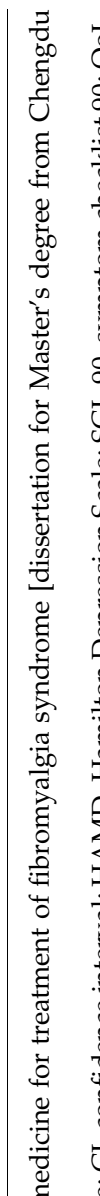

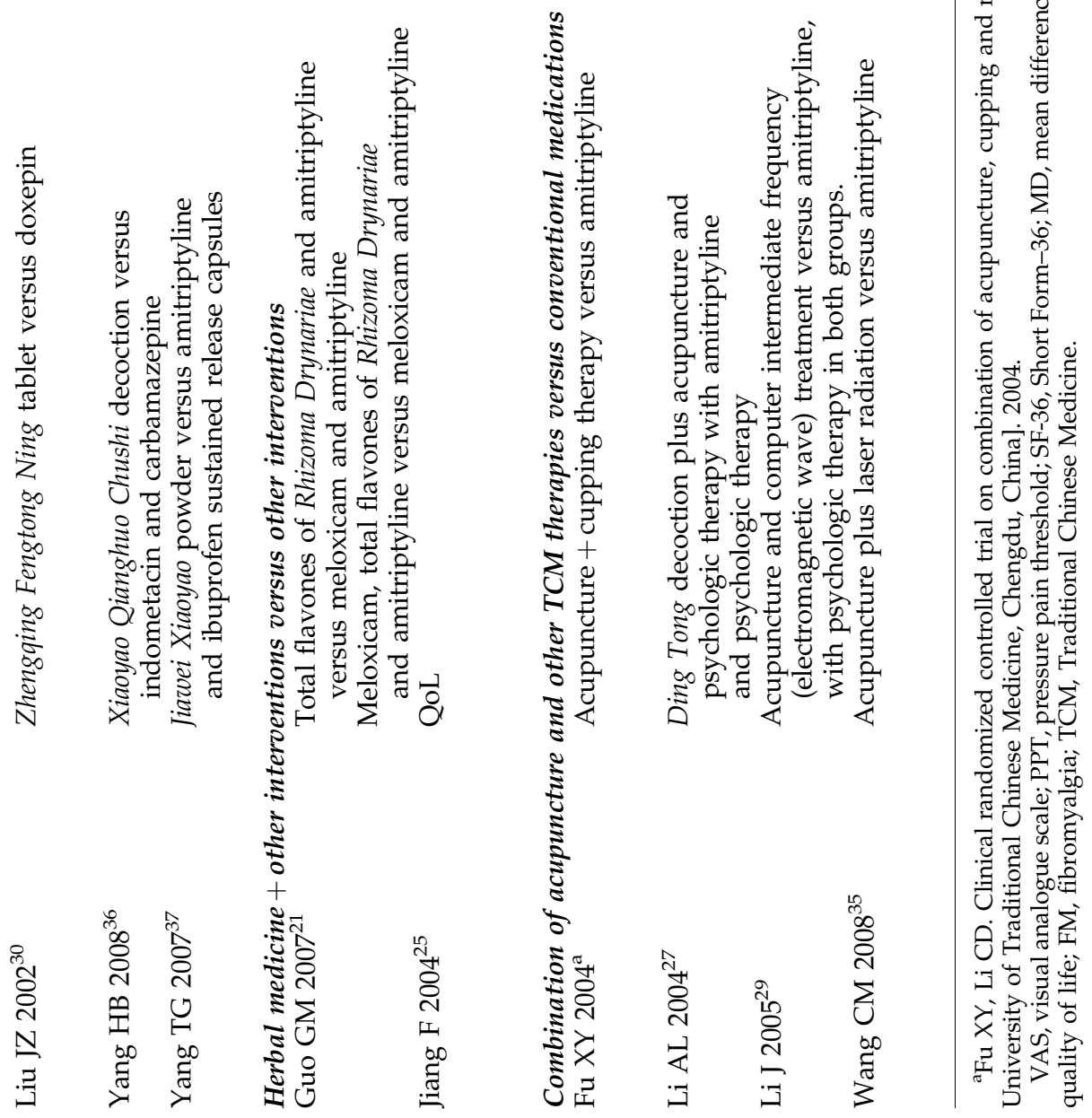


acupuncture or herbal medicine, blinding of outcome assessors should be attempted as far as possible to minimize performance and assessment biases. Choosing outcome measures should be based on an international consensus and should include continuous data and daily average pain scores from baseline to study completion. Analysis of outcomes based on the intention-to-treat principle is vital as is the application of well-defined diagnostic criteria, such as ACR 1990, thus, increasing comparability between trials. Reporting of trials should follow Consolidated Standards of Reporting Trials (CONSORT) ${ }^{52}$ standards to explain the processes involved explicitly and in a transparent manner.

\section{Conclusions}

The preliminary conclusions of the current study suggest that patents with FM might benefit from TCM treatment. FM is a chronic disease and better, larger trials will be the basis for demonstrating the effectiveness and long-term effects of TCM therapies.

\section{Disclosure Statement}

No competing financial conflicts exist.

\section{Acknowledgments}

H.J .Cao and Liu JP were supported by a grant from the National Basic Research Program of China ("973" Program, No. 2006CB504602) and an international cooperation project from the Ministry of Science and Technology of China (2009DFA31460). Liu JP was in part supported by the Grant Number R24 AT001293 from the National Center for Complementary and Alternative Medicine (NCCAM) of the U.S. National Institutes of Health. Lewith GT was supported by the Rufford Maurice Laing Foundation.

\section{References}

1. Russell IJ, Raphael KG. Fibromyalgia syndrome: Presentation, diagnosis, differential diagnosis, and vulnerability. CNS Spectr 2008;13(3suppl 5):6-11.

2. Wolfe F, Smythe HA, Yunus MB, et al. The American College of Rheumatology 1990 Criteria for the Classification of Fibromyalgia: Report of the Multicenter Criteria Committee. Arthritis Rheum 1990;33:160-172.

3. Miller LJ, Kubes KL, Serotonogenic agents in the treatment of fibromyalgia syndrome. Ann Pharmacother 2002;36:707712.

4. Argoff CE. Pharmacologic management of chronic pain. J Am Osteopathic Assoc 2002;102(9suppl3):521-526.

5. Hauser W, Bernardy K, Ueeyler N, Sommer C. Treatment of fibromyalgia syndrome with antidepressants: A metaanalysis. JAMA 2009;301:198-209.

6. Uceyler N, Hauser W, Sommer C. A systematic review on the effectiveness of treatment with antidepressants in fibromyalgia syndrome. Arthritis Rheum 2008;59:1279-1298.

7. Tofferi JK, Jackson JL, O'Malley PG. Treatment of fibromyalgia with cyclobenzaprine: A meta-analysis. Arthritis Rheum 2004;51:9-13.

8. Moore RA, Straube S, Wiffen PJ, et al. Pregabalin for acute and chronic pain in adults. Cochrane Database Syst Rev 2009;3:CD007076.
9. Sim J, Adams N. Systematic review of randomized controlled trials of nonpharmacological interventions for fibromyalgia. Clin J P2002;18:324-336;

10. Fu XY, Li Y, Yang JJ. A survey of acupuncture for fibromyalgia syndrome. Shanghai J Acupunct Moxibustion 2004;237:46-48.

11. U.S. Department of Health and Human Services Public Health Service. Acupuncture for Fibromyalgia. Maryland: U.S. Department of Health and Human Services Public Health Service, Agency for Healthcare Research and Quality, 2003.

12. Mayhew E, Ernst E. Acupuncture for fibromyalgia-a systematic review of randomized clinical trials. Rheumatology 2007;46:201-204.

13. Wahner-Roedler DL, Elkin PL, Vincent A, Thompson JM, et al. Use of complementary and alternative medical therapies by patients referred to a fibromyalgia treatment program at a tertiary care center. Mayo Clin Proc 2005;80:55-60.

14. Higgins JPT, Green S, eds. Cochrane Handbook for Systematic Reviews of Interventions, Version 5.0.1 [updated September 2008]. The Cochrane Collaboration, 2008. Online document at: www.cochrane-handbook.org Accessed March 27, 2010.

15. Higgins JP, Thompson SG. Quantifying heterogeneity in a meta-analysis. Statistics Med 2002;21:1539-1558.

16. Assefi NP, Sherman KJ, Jacobsen C, Goldberg J, Smith WR, Buchwald D. A randomized clinical trial of acupuncture compared with sham acupuncture in fibromyalgia. Ann Intern Med 2005;143:10-19.

17. Brattberg G. Connective tissue massage in the treatment of fibromyalgia. Eur J Pain 1999;3:235-245.

18. Cao JY, Li Y. Combination of acupuncture and antidepressant medications in treating of 56 cases of fibromyalgia. Chin Arch Trad Chi Med 2003;21:813-817.

19. Deluze C, Bosia L, Zirbs A, et al. Electroacupuncture in fibromyalgia: Results of a controlled trial. BMJ 1992;305:1249-1252.

20. Fu HW, Jin ML, Hong J. Herbal decoction with function of relieving $q i$ stagnancy in liver and promoting Blood circulation to remove meridian obstruction in treating 21 cases of fibromyalgia. Zhejiang J Trad Chin Med 2006;41:204-205

21. Gao GM, Jiang L, Liu SY, et al. A randomized and controlled study of clinical efficacy for total flavone of Rhizoma Drynariae on fibromyalgia syndrome. Chin J New Drug Clin Remedies 2007;26:837-840.

22. Guo XJ, Jia J. Comparison of therapeutic effects of transcutaneous electrical nerve stimulation and electroacupuncture on fibromyalgia syndrome. Chin Acupunct Moxibustion 2003;23:653-655.

23. Guo Y, Sun YZ. Clinical study on treatment of fibromyalgia syndrome with penetration needling at the back. ChinAcupunct Moxibustion 2005;25:98-100.

24. Harris RE, Tian X, Williams DA, et al. Treatment of fibromyalgia with formula acupuncture: Investigation of needle placement, needle stimulation, and treatment frequency. J Altern Complement Med 2005;11:663-671.

25. Jiang F, Liu JB. An observation on clinical efficacy in fibromyalgia syndrome. Chin J Rheumatol 2004;8:750-752.

26. Lautenschlager J, Schnorrenberger CC, Muller W. Acupuncture with general fibromyalgia syndrome. Dtsch Zschr Akup 1989;32:122-128.

27. Li AL, Wang ZL, Ping Y. Clinical observation of the treatment of primary fibrositis syndrome by Ding Tongtang combined with acupuncture. Chin J Curr Clin Medic 2004;2(9A):1387-1388.

28. Li CD, Fu XY, Jiang ZY, et al. Clinical study on combination of acupuncture, cupping and medicine for treatment of 
fibromyalgia syndrome. Chin Acupunct Moxibustion 2006; 26:8-10.

29. Li J, Yu HB, She T. The clinical effect of acupuncture and computer [sic] intermediate frequency for fibromyalgia syndrome. Modern Med J China. 2005;7:42-44.

30. Liu JZ, Liu YF, Liu JJ. Clinical observation of Zhengqing Fengtong Ning tablet for fibromyalgia syndrome. Chin J Integr Med 2002;22:316.

31. Liu Q, Li F. Clinical observation of acupuncture for 30 cases of fibromyalgia. Anthol Med 2002;21:183.

32. Martin DP, Sletten CD, Williams BA, Berger IH. Improvement in fibromyalgia symptoms with acupuncture: Results of a randomized controlled trial. Mayo Clin Proc 2006;81:749-757.

33. Sprott H. Efficiency of acupuncture in patients with fibromyalgia. Clin Bull Myofascial Ther 1998;3:37-43.

34. Targino RA, Imamura $\mathrm{M}$, Kaziyama $\mathrm{HH}$, et al. A randomized controlled trial of acupuncture added to usual treatment for fibromyalgia. J Rehabil Med 2008;40:582-588.

35. Wang CM. Combination of acupuncture and semiconductor laser in treating 28 cases of fibromyalgia syndrome. J Practical Trad Chin Intern Med 2008;22:58.

36. Yang HB. Combination of Xiaoyao Qianghuo Chushi decoction and Suanzao Mugua Sanqi Medicinal Liquor in treating 38 cases of fibromyalgia syndrome. J Clin Exper Med 2008; 7:146-147.

37. Yang TG, Feng XH. Clinical study on Jiaweixiaoyao powder [sic] treat the fibromyalgia syndrome. Liaoning J Trad Chin Med 2001;20:438.

38. Zhang YG. Clinical observation on acupuncture treatment of primary fibromyalgia syndrome. Zhongguo Zhen Jiu 2008; 8:7619-7620.

39. Guo XJ, Jia J. Clinical observation of therapeutic effects of transcutaneous electrical nerve stimulation on fibromyalgia syndrome. Clin J Phys Med Rehabil 2004;26:126-127.

40. Guo XJ, Jia J. Comparison of therapeutic effects of transcutaneous electrical nerve stimulation and electroacupuncture on fibromyalgia syndrome. Chin J Clin Rehabil 2005;9:171-173.

41. Chen HJ, Wang GF, Gao M. Medium frequency electrotherapy combined with semiconductor laser in treating with psoas fibrositis. Chin J Phys Med Rehabil 2004;26:739.

42. Liang Q. Combination of acupuncture with warm needle and cupping therapy in treating of 42 cases of fibrositis. J Practical Tradit Chin Intern Med 1999;13:50.
43. Liu LJ. Combination of electroacupuncture and cupping therapy on treatment of 40 cases of fibrositis. Chin Acupunct Moxibustion 1995;20.

44. Ma TW, Pei JZ. Combination of massage and moxibustion in treating 57 cases of shoulder fibrositis. Chin Manipulation Qi Gong Ther 2003;19:23.

45. Wang LQ. Combination of cupping and auricular therapy in treating 60 cases of fibrositis. Shandong J Tradit Chin Med 1996;15:24.

46. Wang SP, Wang XF, Zhang DX, et al. Clinical observation on therapeutic effect of acupuncture treatment based on syndrome differentiation of meridians on fibromyalgia syndrome. Chin Acupunct Moxibustion 2002;22:807-809.

47. Yang NH, Wang W. Combination of herbal tincture and TDP irradiation for fibrositis. Herald Med 2001;20:438.

48. Zuo YM. Clinical observation of Jinbitong capsule for fibrositis. Clin J Misdiagnostics 2008;8:7619-620.

49. Ronald K. Pain Management Secrets. Beijing: China Ocean Press, 1999:149.

50. Martin-Sanchez E, Torralba E, Diaz DE, et al. Efficacy of acupuncture for the treatment of fibromyalgia: Systematic review and meta-analysis of randomized trials. Open Rheumatol J 2009;3:25-29.

51. Madsen MV, Gotzsche PC, Hrobjartsson A. Acupuncture treatment for pain: Systematic review of randomized clinical trials with acupuncture, placebo acupuncture and no acupuncture groups. BMJ 2009;338:a3115 doi:10.1136/bmj .a3115;

52. CONSORT Statement 2001-Checklist: Items to Include When Reporting a Randomized trial. Online document at: www.consort-statement.org Accessed Aug 31, 2009.

Address correspondence to: Jianping Liu, Ph.D., M.D.

Centre for Evidence-Based Chinese Medicine Beijing University of Chinese Medicine 11 Bei San Huan Dong Lu Chaoyang District 1000029, Beijing China

E-mail: Jianping_1@hotmail.com 
